Electrophoretic NMR characterisation of charged sidechain cationic polyelectrolytes and their interaction with the anionic surfactant, sodium dodecylsulfate

Leesa Patel ${ }^{1}$, Omar Mansour $^{1}$, Martin Crossman ${ }^{2}$ and Peter Griffiths ${ }^{1,{ }^{*}}$

Supplemental Section

(15 pages, 9 figures and 1 table) 


\section{PGSE-NMR Analysis}

In a PGSE-NMR experiment, one records an NMR spectrum as a function of increasing field gradient strength. The key information is contained in the rate of decay of the chemically distinct peaks. In a CORE analysis, one presents the original dataset "as recorded" in a 3D format, with the spectrum running left to right, with increasing field gradient intensity increasing front to back.

In the CORE analysis, one is able to "mask" or edit out specific peaks or regions of the spectrum, and this is routinely carried out for the water peak. The CORE analysis then seeks a global minimum for the entire dataset, given a set of input criteria, viz a measure of the signal-to-noise, the number of components present (and hence, the number of expected diffusion coefficients) that best describe the attenuation of all of the points in each of the peaks. A key output from this analysis is the residuals map, being the difference between the fit to (or simulation of) the data, and the original dataset. An appropriate fit in this representation, would be manifest as a small absolute magnitude of noise, and an absence of any coherent waves or significant intensity across regions of the 3D map.

From the figures below, it is clear that when one analyses just the SDS part of the spectrum, a simple single exponential yields a very good representation of those attenuation functions (figure S1.1(a)(c)). For the polymer specific peaks, a single exponential is does not represent a good fit to the data, with there being a coherent wave in the residuals map, representative of an inappropriate model (figure S1.2(b)). Normally, this is interpreted as the polymer having slight polydispersity, and one uses a stretched exponential analysis as a simple way of representing a range of molecular weight. (This is akin to the cummulants method used in dynamic light scattering). As may be seen, this model provides a very satisfactory representation of the data figure S1.2(c)), and thus, it is this analysis that has been carried out for all of the polymers mentioned here.

Thus, the global dataset is described by a two component fit, characterised by two diffusion coefficients, and a parameter that captures the width of the distribution of diffusive rates present. 

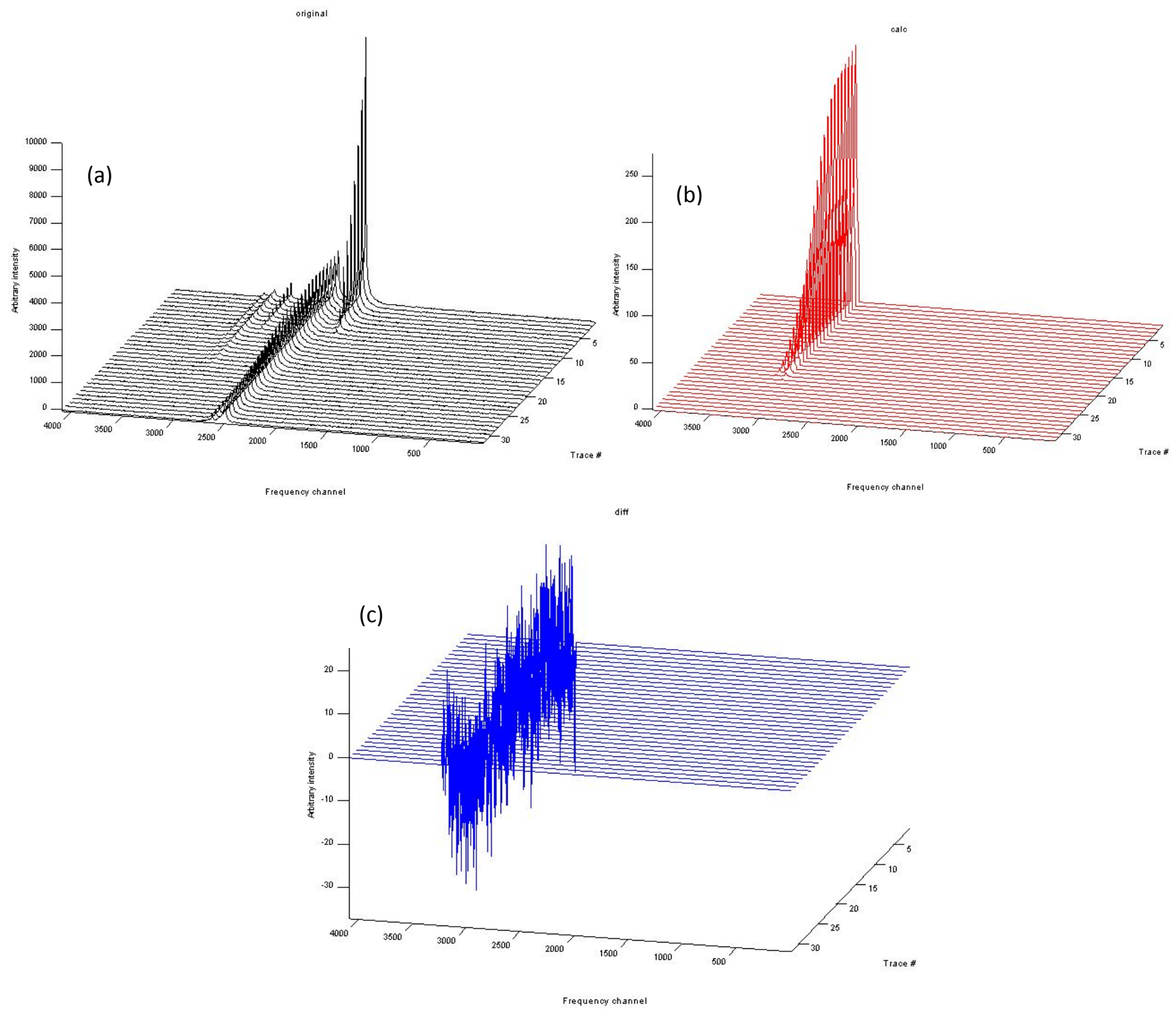

Figure S1.1. CORE analysis of an aqueous solution of $1 \mathrm{wt} \% \mathrm{~N}=0.9 \%$ and $4 \mathrm{mM}$ SDS. Here the experiment has been optimised for the SDS diffusion, showing (a) the original data, (b) fit of the solvent \& polymer masked spectrum to a single exponential model, and (c) residuals. Note the relative y-scales. 

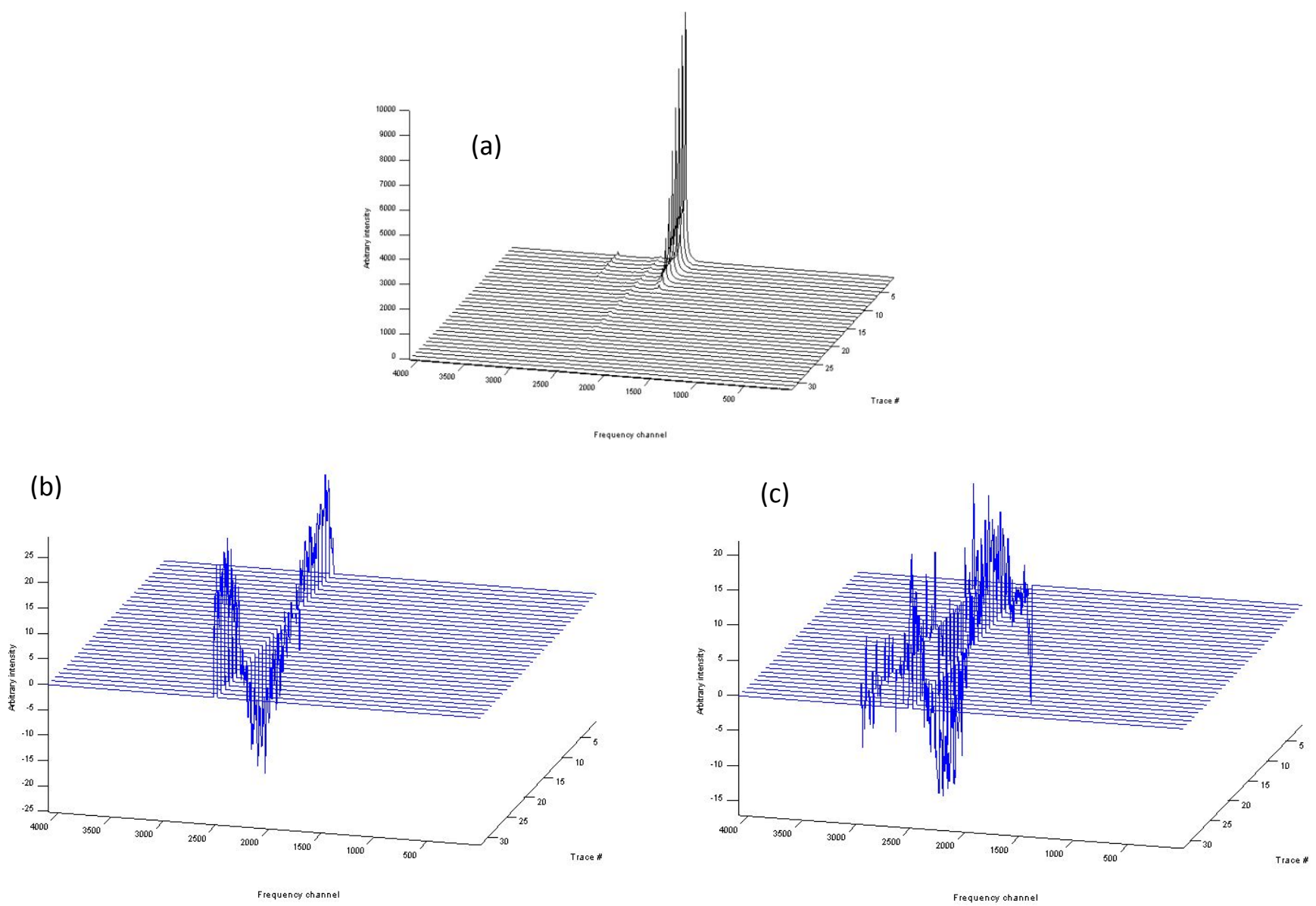

(d)

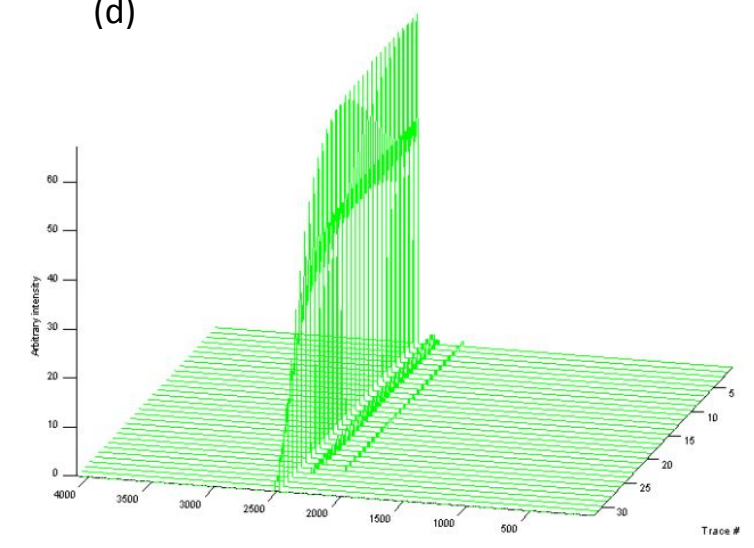

Figure S1.2: CORE analysis of an aqueous solution of $1 \mathrm{wt} \% \mathrm{~N}=0.9 \%$ and $4 \mathrm{mM}$ SDS. Here the experiment has been optimised for the polymer diffusion, showing (a) the original data, (b) residuals from single exponential (monodisperse) model, (c) residuals for stretched exponential (polydisperse) model, and (d) polymer component. Note the relative y-scales.

Once the individual self-diffusion coefficients of the polymer $D_{S}^{\text {poly }}$ and the surfactant have been determined, it is possible to calculate the time the surfactant spends in either of the two limiting environments (1) free in solution, $p_{\text {free }}$ where it diffuses at the same rate $D_{s}^{\text {unimer }}$ as a dilute, pre-CMC 
solution, or (2) bound to the polymer, $p_{\text {bound, }}$ where it will diffuse at the same rate as the polymer. Time-averaging and fast exchange between the two environments leads to an average value of the surfactant self-diffusion coefficient $\left\langle D_{S}^{\text {surf }}\right\rangle$ that reflects the distribution of the surfactant between these two states, viz

$\left\langle D_{s}^{\text {surf }}\right\rangle=p_{\text {free }} D_{s}^{\text {unimer }}+p_{\text {bound }} D_{S}^{\text {poly }}$

where $p_{\text {free }}+p_{\text {bound }}=1$. Hence, $p_{\text {free }}=\frac{\left(\left\langle D_{s}^{\text {surf }}\right\rangle-D_{s}^{\text {poly }}\right)}{\left(D_{s}^{\text {unimer }}-D_{s}^{\text {poly }}\right)}$, from which the free, non-adsorbed concentration may be calculated since $p_{\text {free }}=c_{\text {free }} / c_{\text {total }}$.

\section{eNMR analysis}

e-NMR is a method that combines electrophoresis with diffusion NMR. Electrophoresis is the transport of charged particles in an electric field; under the effect of a constant electric field Edc, lons of a particular species attain the steady state velocity;

$v e=\mu E d c$

where $\mu$ is the electrophoretic mobility.

The technique is a combination of diffusion and electrophoresis. Two platinum wire electrodes are inserted into $5 \mathrm{~mm}$ NMR tubes to generate the electric field that is responsible for the resulting plug flow along the z-axis, leading to a phase shift in the spectrum. The experiment is usually set up such that positive species gives positive phase shift and negative species gives a negative phase shift, but this is just convention.

An electrophoretic double stimulated echo is used in which the direction of the two electrophoretic pulses are switched after half the diffusion time. This has the advantage of suppressing artefacts in the phase shift due to thermal convection. Further, quantifying the movement of uncharged species, these can be used as a reference to check for bulk flow. By subtracting the phase shift of the charged compound from that of the uncharged one, the true electrophoretic phase shift can be derived. Plotting that phase shift as a function of applied voltage, yields the electrophoretic mobility, according to the following equation;

$\mu=\frac{\mathrm{l} \emptyset}{\gamma g \delta \Delta \mathrm{U}}$ 
where $\mu$ is electrophoretic mobility in $\left[\mathrm{m}^{2} \mathrm{~V}^{-1} \mathrm{~s}^{-1}\right], l$ is the distance between the two electrodes in $[\mathrm{m}]$, $\gamma$ is the gyromagnetic ratio in $\left[\mathrm{s}^{-1} \mathrm{~T}^{-1}\right], \mathrm{g}$ is the magnetic field gradient in $\left[\mathrm{Tm}^{-1}\right], \delta$ diffusion time $[\mathrm{s}], \Delta$ is the duration of the gradient pulse in [s], $\emptyset$ is the phase shift in [rad] and $U$ the applied voltage in [V].

Detecting phase (instead of integral intensity) is advantageous because NMR signal phase modulation is clearly separable from NMR signal attenuation, a distinction that is not possible when recording NMR cosine modulation observed in a U-tube arrangement. This has the impact of being able to very easily obviate distortion to the signal integral intensity arising from thermal convection, electro osmosis, bubble formation.

Thus, the phase correction method combined with convection compensated pulse sequences provides a path to accurate electrophoretic mobility data. This experimental approach requires having uncharged molecule contained within the sample, and water is particularly useful in this regards.

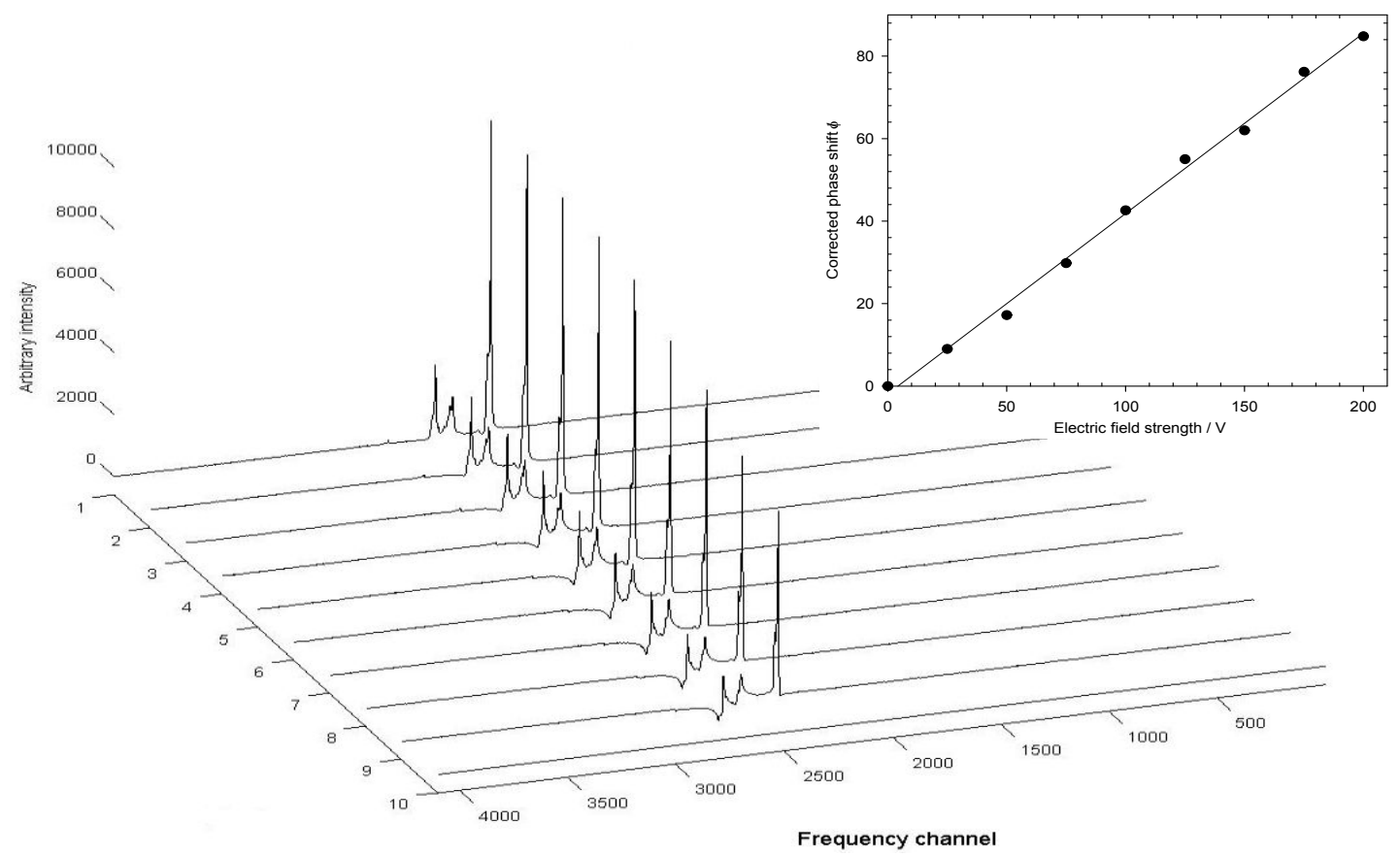

Figure S2. (main) Experimental electrophoretic-NMR dataset for an aqueous solution of $1 \mathrm{wt} \% \mathrm{~N}=1.6 \%$ polymer, showing (inset) the required phase shift correction for the polymer peaks as a function of electric field parameters. 


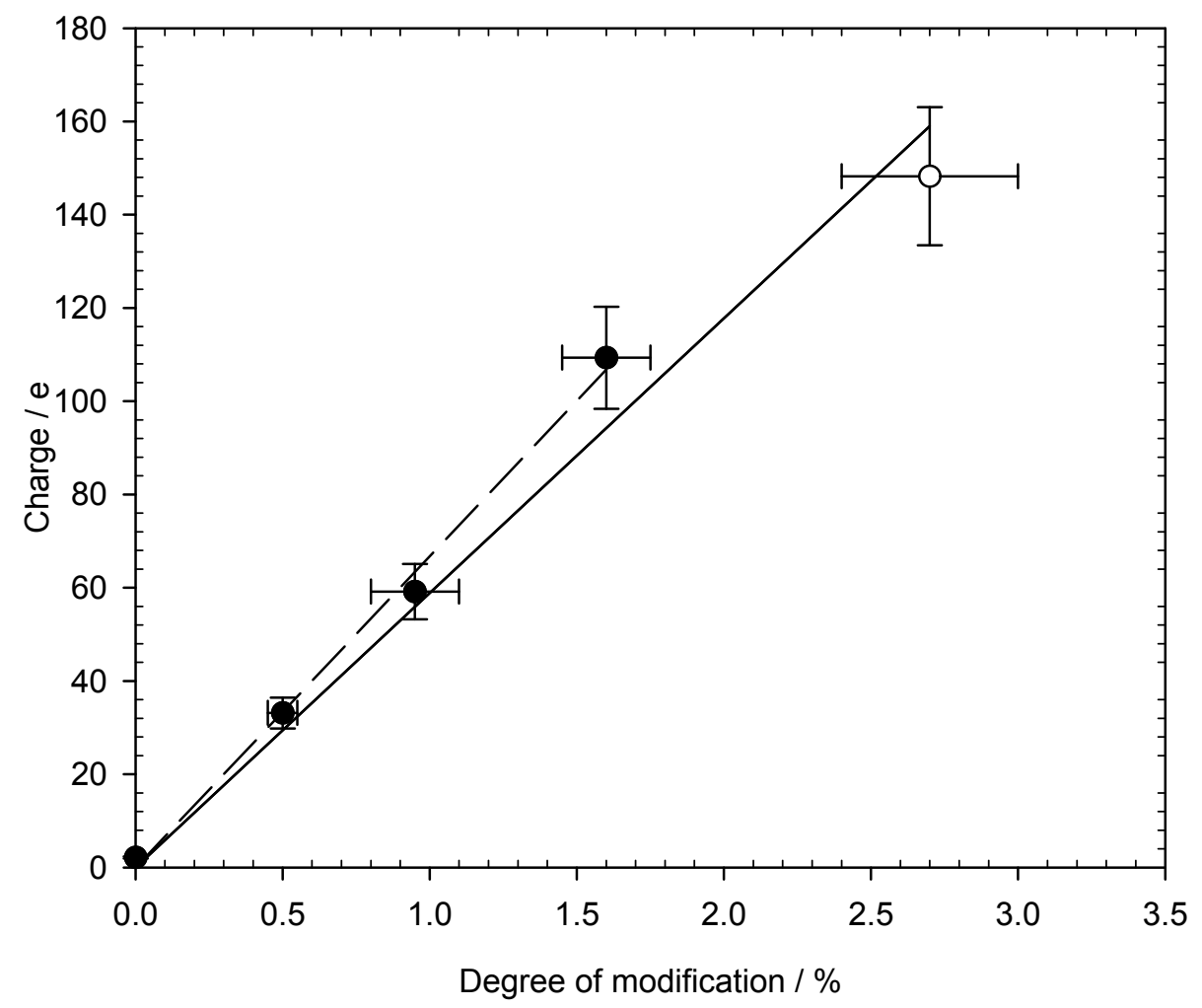

Figure S3. Effective charge on the quaternized hydroxycellulose polymer at infinite dilution as a function of degree of modification. 


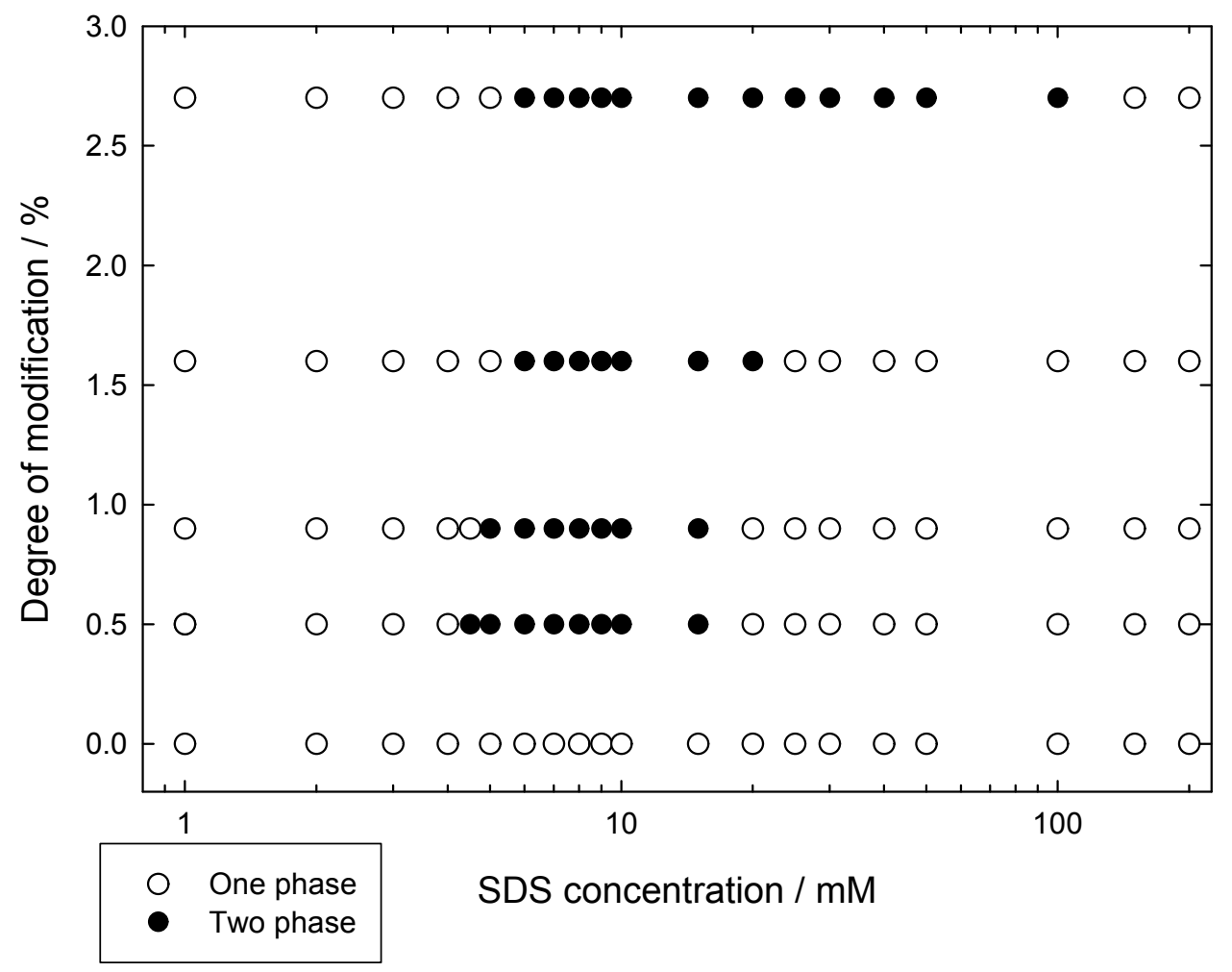

Figure S4. Phase diagram for the quaternized hydroxycellulose polymers studied here at $\mathrm{C}_{\text {polymer }}=1 \mathrm{wt}$ $\%$ according to their degree of modification and SDS concentration. 


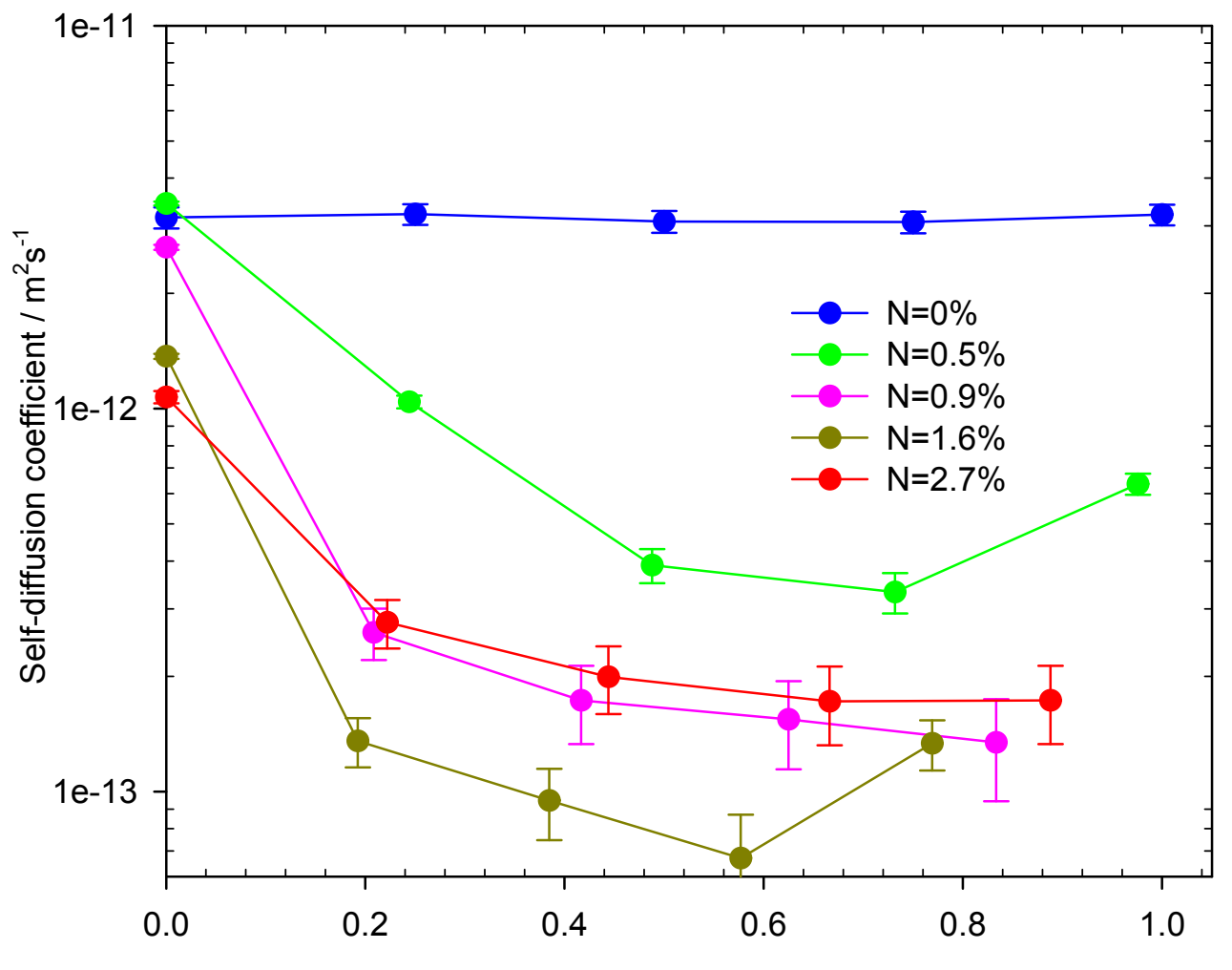

Total SDS conc. normalised to the phase boundary

Figure S5. Polymer self-diffusion coefficient in the presence of SDS as a function of total SDS concentration normalised by the phase boundary concentration in Figure $\mathrm{S} 4$. For the $\mathrm{N}=0 \%$ case, the data are plotted in terms of the total SDS concentration as no phase boundary was observed. 


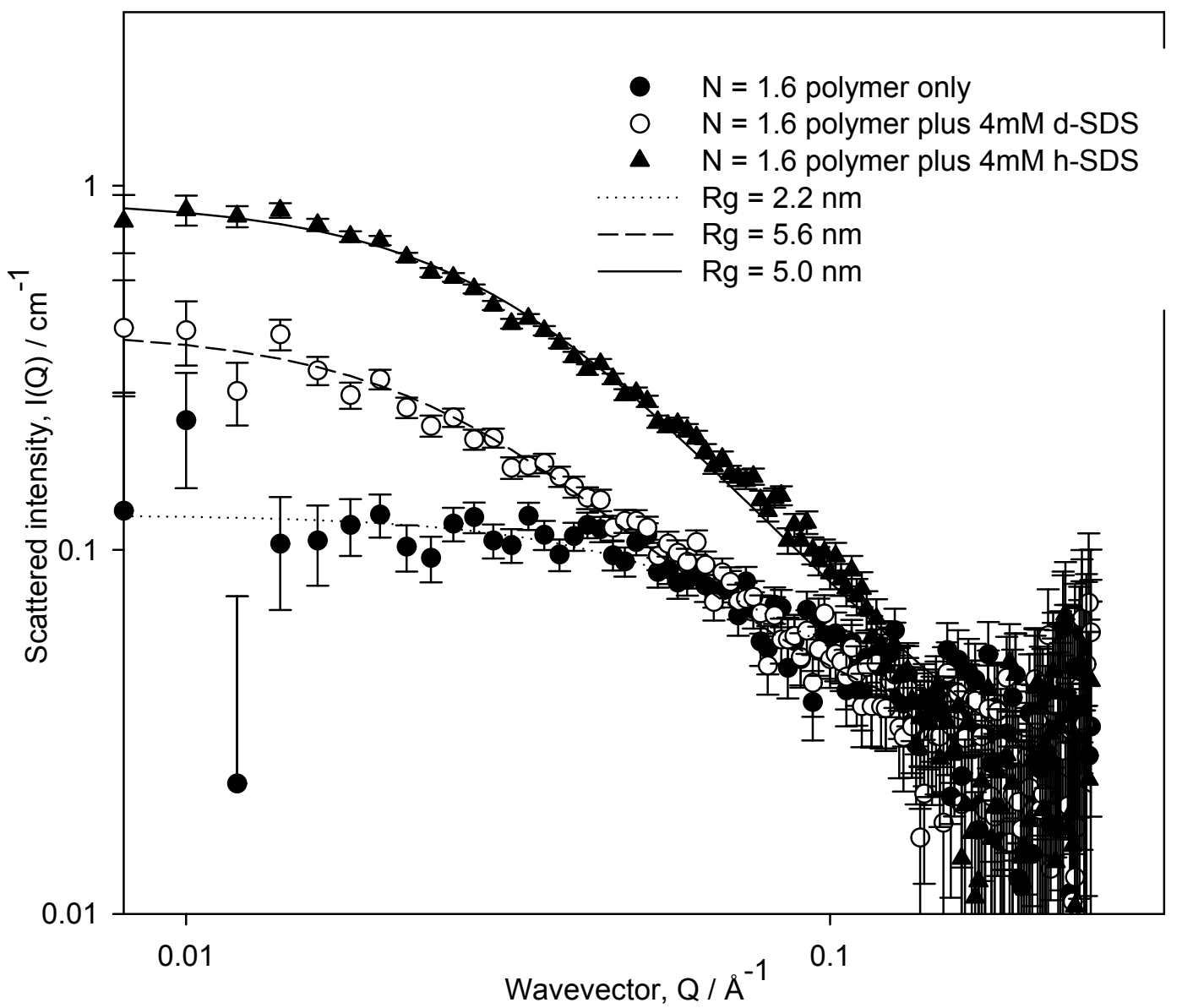

Figure S6. Small-angle neutron scattering from $1 \mathrm{wt} \%$ aqueous solutions of $\mathrm{N}=1.6 \%$ polymer in the absence and presence of $4 \mathrm{mM}$ SDS, the latter for both deuterated and hydrogenous forms.

Figure $\mathrm{S} 6$ shows scattering data from $1 \mathrm{wt} \%$ aqueous $\mathrm{N}=1.6 \%$ polymer solutions. The scattering is weak, as might be expected given a polymer of this nature and its poor contrast, but the polymer-only data shows a very gradual decay of intensity vs. wavevector, which is consistent with a Gaussian coil with radius of gyration, $R_{\mathrm{g}}=2.2 \mathrm{~nm}$.

On addition of SDS, both deuterated and hydrogenated, there is an increase in the scattering, which is more marked in the presence of the hydrogenous surfactant, again as might be expected. For the d-surfactant case, in $\mathrm{D}_{2} \mathrm{O}$, the surfactant is said to be "contrast matched", and does not contribute to the measured scattering. The two curves - polymer and polymer/d-SDS - are largely similar, suggesting that the polymer and the polymer/surfactant complex have the same gross shape. Both decays may be described by a Gaussian conformation, consistent with the same radius of gyration, $R_{g}=5.0 \mathrm{~nm}$ or $5.6 \mathrm{~nm}$ 


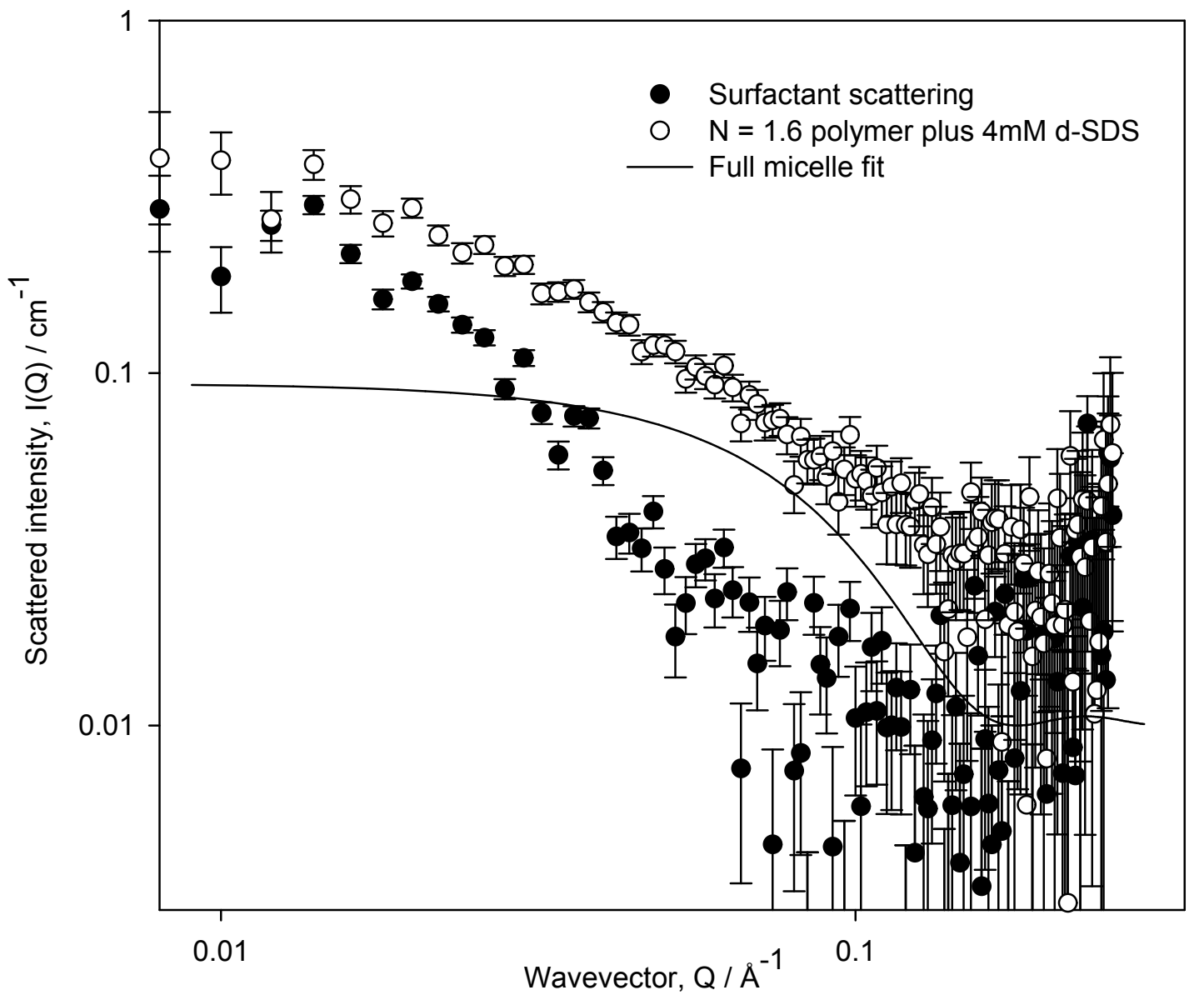

Figure S7. Difference in small-angle neutron scattering from $1 \mathrm{wt} \%$ aqueous solutions of $N=1.6 \%$ polymer in the presence of $4 \mathrm{mM}$ SDS hydrogenous SDS ("surfactant" scattering) and the same system with the deuterated surfactant. To a first approximation, the difference may be regarded as the additional scattering arising from the surfactant itself. The solid line represents the scattering expected from a dilute dispersion of solid objects with dimensions consistent with an SDS micelle with an aggregation number, $\mathrm{N}_{\text {agg }}=55$.

The difference in the scattering of the hydrogenous and deuterated cases -Figure S7 - can be crudely interpreted as the scattering arising from the surfactant alone (but in the presence of the polymer) and shows no characteristic of fully-grown micellar scattering. One can conclude from this very limited set of data, that on binding of the anionic surfactant to the cationic polymer, there is an increase in the dimension characterising the polymer conformation, and that the state of the bound surfactant molecules are not consistent with a fully-grown micellar structure, with aggregation number of 55 . 
Further insight into the state of the bound SDS may be gained from the spectral characteristics of a solubilized probe, and pyrene is a common experimental approach. The ratio of the first to third vibronic peaks $(\mathrm{I} / \mathrm{III})$ is a signature of the polarity sensed by the pyrene, with a micellar environment $(\mathrm{I} / \mathrm{III} \approx 0.8)$ being somewhat different to the aqueous one $(\mathrm{I} / \mathrm{III} \approx 1.3)$.

\begin{tabular}{|c|c|}
\hline System & I / III ratio \\
\hline $4 \mathrm{mM}$ SDS & $1.35( \pm 0.05)$ \\
\hline $0.5 \mathrm{wt} \% \mathrm{~N}=0$ polymer, 2mM SDS & $1.60( \pm 0.10)$ \\
\hline $0.5 \mathrm{wt} \% \mathrm{~N}=0.9$ polymer, 2mM SDS & $1.02( \pm 0.04)$ \\
\hline $0.5 \mathrm{wt} \% \mathrm{~N}=1.6$ polymer, 2mM SDS & $1.11( \pm 0.08)$ \\
\hline $0.5 \mathrm{wt} \% \mathrm{~N}=2.7$ polymer, 2mM SDS & $1.06( \pm 0.03)$ \\
\hline
\end{tabular}

Table S1. I/III ratios for various selected polymer/SDS combinations approaching the phase boundary.

Table S1 displays the $1 /$ III ratios for key samples in this study, albeit at a slightly lower total concentration compared to the NMR studies (to facilitate sample handing of these viscous solutions), but at corresponding points on the phase diagram. The $4 \mathrm{mM}$ SDS sample is below the CMC, so no micelles are to be expected, as reflected in the measured I/III ration. Similarly, the $\mathrm{N}=0$ polymer in the presence of $2 \mathrm{mM}$ SDS also has a high $\mathrm{I} / \mathrm{III}$ ration indicating the absence of any micellar environment, consistent with the NMR diffusion analysis. By contrast, there are marked reductions in the I/III ratios for the $\mathrm{N}=0.9,1.6$ and 2.7 polymers with $2 \mathrm{mM}$ SDS, indicative of a more hydrophobic environment, again consistent with the NMR analysis. The values of these ratios suggest that the hydrophobic environment is slightly more polar than a typical SDS micelle, implying an aggregation number less than 55, consistent with the (limited) scattering data in Figure S7. 


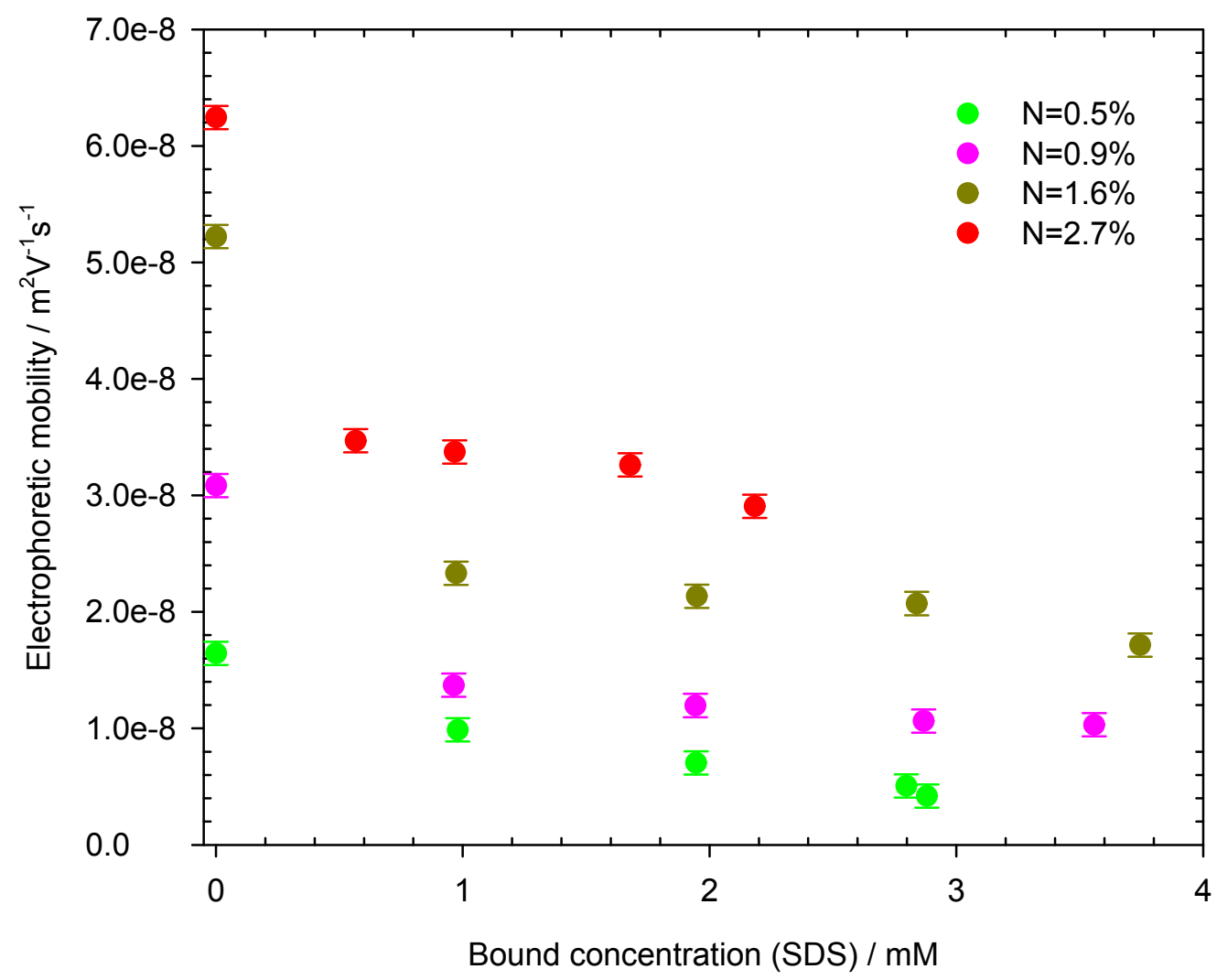

Figure S8. Electrophoretic mobility of the polymer in polymer-SDS blends as a function of polymerbound SDS concentration, for the series of differing degrees of modification; $C_{\text {polymer }}=1 \mathrm{wt} \%$ in $\mathrm{D}_{2} \mathrm{O}$ at $25^{\circ} \mathrm{C}$. 


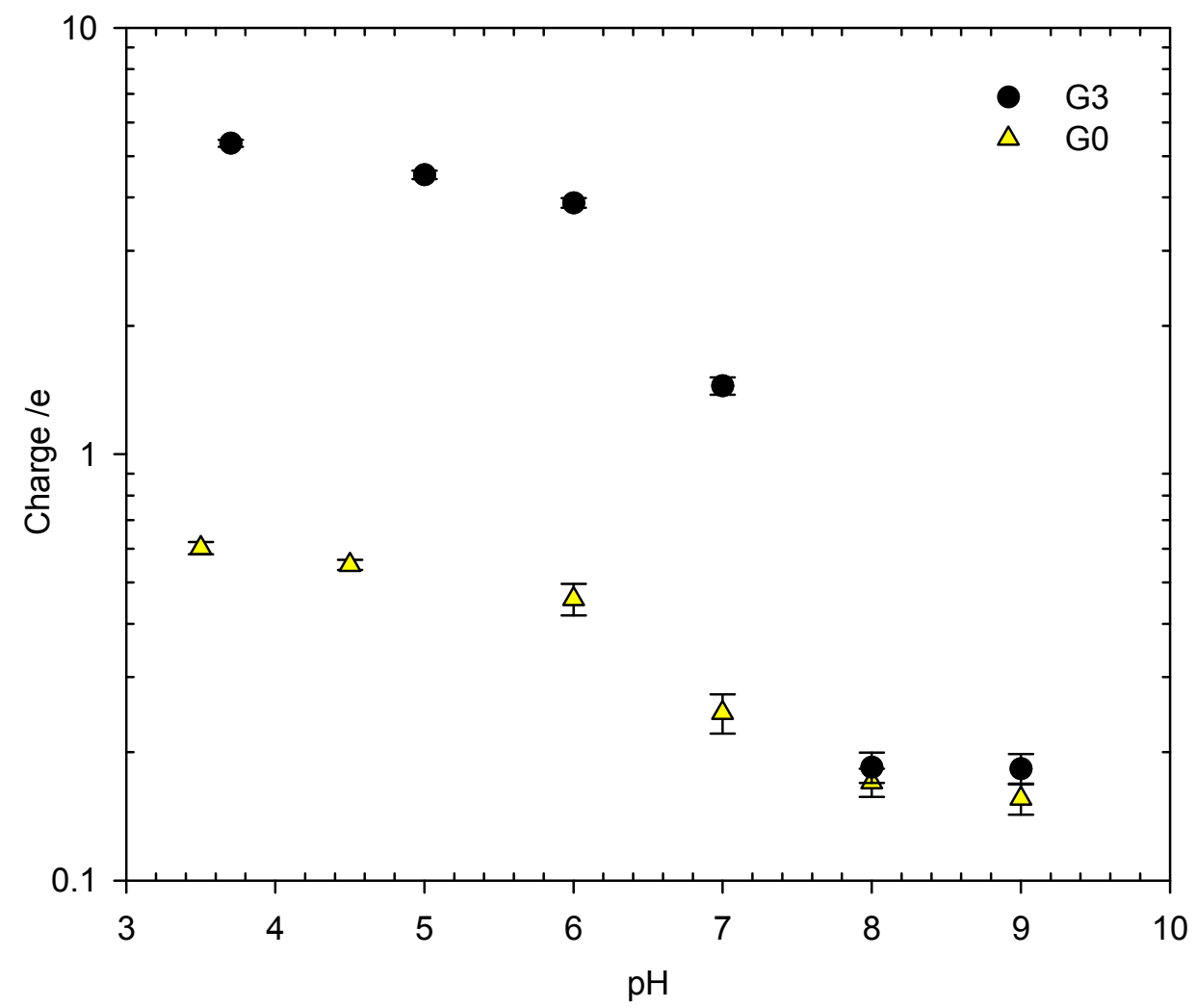

Figure S9. Effective charge on PAMAM G0 and PAMAM G3 in aqueous solutions as a function of $\mathrm{pH}$, at $25^{\circ} \mathrm{C}$.

As with the eNMR analysis of the polymers, the charge determined from the combined diffusion-electrophoretic mobility analysis seems to "under report" slightly the absolute charge on these small polymers - PAMAM GO has 4 surface charges, but eNMR reports a charge of $0.6 \mathrm{e}$, whereas for PAMAM G3, the respective numbers are 32 and $5 \mathrm{e}$. Interestingly, the ratio of the two pairs of number ( 8 and 8.5 resp.) suggests that eNMR is appropriately detecting the relative changes. 